\title{
Sex- and tissue-specific expression of odorant-binding proteins and chemosensory proteins in adults of the scarab beetle Hylamorpha elegans (Burmeister) (Coleoptera: Scarabaeidae)
}

Angélica González-González ${ }^{1,2}$, María E. Rubio-Meléndez ${ }^{3}$, Gabriel I. Ballesteros ${ }^{1,2}$, Claudio C. Ramírez ${ }^{1,2}$, Rubén Palma-Millanao ${ }^{\text {Corresp. 1,2 }}$

${ }^{1}$ Centre in Molecular and Functional Ecology, Universidad de Talca, Talca, Chile

2 Instituto de Ciencias Biológicas, Universidad de Talca, Talca, Chile

${ }^{3}$ Centro de Bioinformática y Simulación Molecular (CBSM), Facultad de Ingeniería, Universidad de Talca, Talca, Maule, Chile

Corresponding Author: Rubén Palma-Millanao

Email address: rupalma@utalca.cl

In this study, we addressed the sex- and tissue-specific expression patterns of odorantbinding proteins (OBPs) and chemosensory proteins (CSPs) in Hylamorpha elegans (Burmeister), an important native scarab beetle pest species from Chile. Similar to other members of its family, this scarab beetle exhibit habits that make difficult to control the pest by conventional methods. Hence, alternative ways to manage the pest populations based on chemical communication and signaling (such as disrupting mating or host finding process) are highly desirable. However, developing pest-control methods based on chemical communication requires to understand the molecular basis for pheromone recognition/chemical perception in this species. Thus, with the aim of discovering olfactionrelated genes, we obtained the first reference transcriptome assembly of $\mathrm{H}$. elegans. We used different tissues of adult beetles from males and females: antennae and maxillary palps, which are well known for embedded sensory organs. Then, the expression of predicted odorant-binding proteins (OBPs) and chemosensory proteins (CSPs) was analyzed by qRT-PCR. In total, 165 transcripts related to chemoperception were predicted. Of these, 16 OBPs, including one pheromone-binding protein (PBP), and four CSPs were successfully amplified by qRT-PCR. All of these genes were differentially expressed in the sensory tissues with respect to the tibial tissue that was used as a control. The single predicted PBP found was highly expressed in the antennal tissues, particularly in males, while several OBPs and one CSP showed male-biased expression patterns, suggesting that these proteins may participate in sexual recognition process. In addition, a single CSP was expressed at higher levels in female palps than in any other studied condition, suggesting that this CSP would participate in oviposition process. Finally, all four CSPs exhibited palp- 
biased expression while mixed results were obtained for the expression of the OBPs, which were more abundant in the palps than in the antennae. These results suggest that these chemoperception proteins would be interesting novel targets for control of $H$. elegans, thus providing a theoretical basis for further studies involving new pest control methods. 
1 Sex- and tissue-specific expression of odorant-binding proteins and chemosensory proteins in

2 adults of the scarab beetle Hylamorpha elegans (Burmeister) (Coleoptera: Scarabaeidae)

5 Angélica González-González ${ }^{1,2}$, María E. Rubio-Meléndez ${ }^{3}$, Gabriel I. Ballesteros ${ }^{1,2}$, Claudio C.

$8{ }^{1}$ Centre in Molecular and Functional Ecology, Universidad de Talca, Talca, Chile

$9 \quad{ }^{2}$ Instituto de Ciencias Biológicas, Universidad de Talca, Talca, Chile

$10{ }^{3}$ Centro de Bioinformática y Simulación Molecular (CBSM), Facultad de Ingeniería, Universidad

11 de Talca, Talca, Chile

12 *Corresponding author: Rubén Palma-Millanao, Universidad de Talca, Talca, Chile.

13 Tel: +56 712200267. E-mail address: rupalma@utalca.cl 


\section{Abstract}

In this study, we addressed the sex- and tissue-specific expression patterns of odorant-binding proteins (OBPs) and chemosensory proteins (CSPs) in Hylamorpha elegans (Burmeister), an important native scarab beetle pest species from Chile. Similar to other members of its family, this scarab beetle exhibit habits that make difficult to control the pest by conventional methods. Hence, alternative ways to manage the pest populations based on chemical communication and signaling (such as disrupting mating or host finding process) are highly desirable. However, developing pest-control methods based on chemical communication requires to understand the molecular basis for pheromone recognition/chemical perception in this species. Thus, with the aim of discovering olfaction-related genes, we obtained the first reference transcriptome assembly of H. elegans. We used different tissues of adult beetles from males and females: antennae and maxillary palps, which are well known for embedded sensory organs. Then, the expression of predicted odorant-binding proteins (OBPs) and chemosensory proteins (CSPs) was analyzed by qRT-PCR. In total, 165 transcripts related to chemoperception were predicted. Of these, 16 OBPs, including one pheromone-binding protein (PBP), and four CSPs were successfully amplified by qRT-PCR. All of these genes were differentially expressed in the sensory tissues with respect to the tibial tissue that was used as a control. The single predicted PBP found was highly expressed in the antennal tissues, particularly in males, while several OBPs and one CSP showed male-biased expression patterns, suggesting that these proteins may participate in sexual recognition process. In addition, a single CSP was expressed at higher levels in female palps than in any other studied condition, suggesting that this CSP would participate in oviposition process. Finally, all four CSPs exhibited palp-biased expression while mixed results were obtained for the expression of the OBPs, which were more abundant in the palps than in the 
antennae. These results suggest that these chemoperception proteins would be interesting novel targets for control of $H$. elegans, thus providing a theoretical basis for further studies involving new pest control methods.

\section{Introduction}

Chemical perception in insects relies on olfaction and gustation, which enable insects to accomplish important tasks such as mating, host finding and predator avoidance in their environment (Sánchez-Gracia, Vieira \& Rozas, 2009). Physically, these abilities depend on small, specialized organs called sensilla (Mutis et al., 2014), which populate insect's appendices, such as antennae and palps. Sensilla are exposed to chemical stimuli and protect the neuronal dendrites inside (Suh, Bohbot \& Zwiebel, 2014). Between the cuticle and the dendrites is the sensillar hemolymph which contains plenty of soluble proteins that carry chemical odorants (Sánchez-Gracia, Vieira \& Rozas, 2009). The most important insect carrier proteins are chemosensory proteins (CSPs) and odorant-binding proteins (OBPs), both of which are responsible for recognizing (Leal, 2013; Li et al., 2013) and transporting volatiles, typically hydrophobic molecules (Gadenne, Barrozo \& Anton, 2016), from the sensillar pores to the membrane receptors located in the dendrites of the neuron (Pelosi et al., 2018). These proteins are the first step in the cascade of events that compromise the olfactory and gustatory processes (Leal, 2013).

OBPs are small globular proteins (10-14 kDa), typically with acidic isoelectric points and are as abundant as $10 \mathrm{mM}$ in the sensillar hemolymph. They are highly divergent in sequence (Zhu, Zhao \& Yang, 2012) and primarily show six highly-conserved cysteine residues, called 
60

61

62

Classic OBPs, that combine to form three disulfide bonds conferring important functional characteristics (Brito, Moreira \& Melo, 2016). Among the OBPs, there are other forms that have less than six cysteines (typically four or five) called Minus-C OBPs (Spinelli et al., 2012) while those with more than six cysteines are called Plus-C OBPs and those with exactly twelve cysteines are called Dimer OBPs (Zhou et al., 2004). In addition to this classification, OBPs can be distinguished from pheromone-binding proteins (PBPs) based on the type of volatile that they bind; PBPs recognize molecules emitted by conspecific individuals (Mao et al., 2016), with sexual pheromones being the most deeply studied. Finally, general odorant-binding proteins (GOBPs) bind general odorants (Glaser et al., 2013) such as host volatiles. In addition, CSPs are smaller than OBPs $(8-11 \mathrm{kDa})$, and they show only four conserved cysteine residues. However, their sequences are more conserved than those of OBPs (Zhu, Zhao \& Yang, 2012), and phylogenetically, CSPs seem to form a more homogeneous group of proteins (Pelosi et al., 2018).

Another important function of these carrier proteins is to protect odorant molecules from the odorant degrading enzymes (ODEs) found in the sensillar lymph (Zhang, Walker \& Wang, 2015) before the odorant molecules reach the olfactory or gustatory receptors (ORs and GRs, respectively). These receptors belong to a large and diverse superfamily of seventransmembrane-domain receptors (Dahanukar, Hallem \& Carlson, 2005; Iatrou \& Biessmann, 2008) expressed in the dendrites inside the sensilla (Leal, 2014) and trigger signal transmission through the neuron to the primary olfactory center of the brain (Gadenne, Barrozo \& Anton, 2016). Interestingly, ORs form a heterodimeric complex with an odorant receptor coreceptor (ORCo) (Leal, 2013). In contrast to ORs, ORCOs are highly conserved among insect species (Dahanukar, Hallem \& Carlson, 2005); only one representative has been reported for each insect 
83 species (Missbach et al., 2014), and its presence is considered crucial for odorant perception

84 (Stengl \& Funk, 2013; Zhou et al., 2014). Thus, these protein families are notoriously important

85 for insects. However, until now, few studies have addressed the sex- and tissue-specific

86 expression patterns of these proteins, especially in numerous groups of pest species among the

87 coleopterans and particularly in the scarab beetles.

88

89

90

91

92

93

94

95

96

97

98

Hylamorpha elegans (Burmeister) is a widespread beetle species native to Chile and is also present in Andean proximities of Argentina's Neuquén province (Ratcliffe \& Ocampo, 2002). As in other interesting cases reported from this family worldwide, this species has been able to adapt to the changes in its environment resulting from the replacement of native vegetation with exotic cultivated species (Lefort et al., 2014, 2015). Thus, it has become an important pest in pastures, cereal and berry crops and common hazel orchards (Durán, 1951;

Cisternas, 1992, 2002, 2013; Aguilera et al., 1996; Aguilera, Guerrero \& Rebolledo, 2011) and is able to cause severe economic damage (Artigas, 1994). Despite the important environmental changes resulting from the introduction of exotic host plant species, H. elegans still has a close relationship with tree species from the genus Nothofagus. Most adult beetles use the canopy of these trees to congregate, feed and mate, and in some seasons, they cause severe defoliation in Nothofagus forests (Bauerle, Rutherford \& Lanfranco, 1997; Lanfranco et al., 2001). As occurs in other scarab beetle species, due to cryptic position of the larvae in the soil and nocturnal activity of the adults, pest control is difficult using conventional methods, such as the use of insecticides applied to the soil (Jackson \& Klein, 2006). Thus, alternative ways to manage the pest populations are highly desirable. Therefore, controlling the beetles based on ethology such as disrupting mating or host finding appears to be an interesting option in controlling this pest (Quiroz et al., 2007; Venthur et al., 2016). Although the chemical communication of the species 
106 has been studied, and the ability of males to perceive receptive females was reported (Quiroz et

107 al., 2007), very little is known about the molecular basis of H. elegans olfaction and chemical

108 communication. However, a recent report revealed an olfactory protein linked to the ability to

109 perceive volatiles commonly used in chemical communication by Scarabaeidae species (Venthur

110 et al., 2014; González-González et al. 2016), which suggests that a similar mechanism could be

111 involved in chemical communication in H. elegans.

Considering this information, our goals were to identify the repertoire of proteins related

to taste and olfaction in adults of Hylamorpha elegans by performing a transcriptome analysis

and comparing the relative expressions of OBPs and CSPs in different tissues of both sexes; we

hypothesized that OBPs and CSPs are highly expressed in the antennae and palps compared to

their expression in a nonsensory tissue, and there are differential expression patterns of these

transcripts between males and females. To our knowledge, this is the first report on the

identification and characterization of multiple olfactory genes in the scarab beetle H. elegans.

\section{Materials \& Methods}

121 Tissue collection. Males and females of $H$. elegans were collected from the field at the peak of

122 their flight season (Quiroz et al., 2007). Males and females ( $n=120$ of each sex) were separated

123 in fresh plastic tubes and anesthetized using cold ice packs. For each individual, both antennae

124 and maxillary palps in addition to the right hindleg tibia were excised using clean tweezers and

125 immersed in RNAlater Solution (Qiagen, Hilden, Germany) following the manufacturer's

126 instructions to preserve RNA. Then, the samples were stored at $-80^{\circ} \mathrm{C}$ until RNA extraction. 
127 RNA extraction and sequencing. Total RNA was extracted grinding the tissue using sterile,

128 disposable plastic pestles in liquid nitrogen, and using the RNeasy Plant Mini kit (Qiagen,

129 Hilden, Germany) following the manufacturer's instructions for use in RNA sequencing and

130 relative expression RT-qPCR assays. A total of 120 males and 120 females were used for RNA

131 extractions, leading to six samples composed of 240 antennae, 240 maxillary palps and 120 right

132 hindleg tibias from each sex. Total RNA was precipitated using 0.1 volumes of $3 \mathrm{M}$ sodium

133 acetate and 2 volumes of 100\% ethanol and shipped to Macrogen (Seoul, South Korea) for

134 sequencing the six libraries. Polyadenylated mRNA was used for library construction using the

135 TruSeq Stranded Total RNA Sample Preparation kit (Illumina, San Diego, CA, USA) and

136 tagged, pooled and sequenced using an Illumina HiSeq 2000 (2×100 bp, paired-end libraries).

137 Two FASTQ files were created for each sex and tissue. Raw transcriptome data were deposited

138 in NCBI's Sequence Read Archive database under ID: SRP137879.

139 Assembly. Illumina RNA-seq libraries were quality checked with FastQC v0.11.3

140 (www.bioinformatics.babraham.ac.uk/projects/fastqc) to assess the presence of adapters derived

141 from sequencing, overrepresented k-mers, read length and overall read quality scores. All

142 libraries were processed with Trimmomatic v0.35 (Bolger et al., 2014) to remove any remaining

143 TruSeq adapter sequences and to eliminate low quality bases $(\mathrm{Q}<30)$ from reads. After

144 sequence processing, all remaining sequences shorter than $36 \mathrm{bp}$ long were also removed from

145 all datasets. Clean Illumina datasets were pooled in silico by concatenating library files. Before

146 assembly, ribosomal RNA reads were removed by mapping the libraries using Bowtie v1.1.1

147 (Langmead et al., 2009) against a custom rRNA database created from insect ribosomal

148 sequences downloaded from NCBI and keeping unmapped reads. The remaining high-quality

149 reads were de novo assembled with Trinity v2.0.6 (Haas et al., 2013) using default parameters. 
150 Metrics for de novo assembly were obtained with QUAST v2.3 (number of contigs, total length,

151 N50, largest contig and \%GC) (Gurevich et al., 2013), while transcriptome completeness was

152 assessed by benchmarking the assembled transcriptome using BUSCO (Benchmarking Universal

153 Single-Copy Orthologs) v3.0.2b (Simão et al., 2015) using the Insecta, Arthropoda and

154 Endopterygota reference database. To determine whether this transcriptome encoded one or more

155 sets of core genes conserved across a range of insect species, a "completeness score" was

156 calculated (Moreton et al., 2016). A total of 1,658 near-universal single-copy orthologs from

157 insect species were used as reference core genes (available at busco.ezlab.org; Simão et al., 158 2015).

Annotation and functional gene classification. Homology searches of contigs from the assembled de novo transcriptome were performed locally with BLASTx using the NR database (NCBI) as a reference, setting an e-value of 1 e-5 as a threshold. Automatic annotation using gene ontology (GO) terms was performed by loading the nucleotide FASTA file (Trinity transcriptome assembly) together with BLASTx results in XML format into Blast2GO v2.8 (Conesa \& Götz, 2008). We also performed InterPro annotation, GO term assignment, and enzyme code and pathway annotation using the Kyoto Encyclopedia of Genes and Genomes (KEGG) terms integrated into Blast2GO. Successfully annotated transcripts were categorized and assigned to GO terms from different GO categories (molecular function, cellular component and biological process). The final contig annotation table (Fig. S1) was exported from Blast2GO.

170 Putative chemoreception-related protein annotation. Identification of putative H. elegans database of protein sequences of OBPs, CSPs, GRs, ORs, IRs, ORCo, SNMPs, ODEs and PDEs 
173 from 954 insect species, using BLASTx (Altschul et al., 1997). These references, known

174 sequences were downloaded from NCBI (ncbi.nlm.nih.gov) and manually curated to build a non-

175 redundant, reference database. Then, putative annotated transcripts were manually curated in

176 order to eliminate isoforms, duplications and incomplete transcripts. Finally, complete-length

177 sequences of the OBPs and CSPs in H. elegans were identified using the ORF finder tool

178 (https://www.ncbi.nlm.nih.gov/orffinder/) (Bin et al., 2017).

179

180

181

182

183

184

185

186

187

188

189

190

191

192

193

194

195

Phylogeny of OBPs and CSPs. Annotated complete length sequences for predicted OBPs and CSPs based on the amino acid sequences from H. elegans, Anomala corpulenta, Holotrichia oblita, Dendroctonus ponderosae and Tribolium castaneum were aligned using ClustalW (Thompson, Higgins \& Gibson, 1994). Then, maximum-likelihood trees were built using CLC Workbench v7.8.1 (Qiagen, Hilden, Germany) using 1,000 replications.

qRT-PCR relative expression analysis. Total RNA from three groups of males and three groups of females (ca. 40 individuals each) was treated to eliminate genomic DNA contamination using a Turbo DNA-free kit (Thermo Fisher, Vilnius, Lithuania) following the manufacturer's protocol. Then, cDNA was synthetized using an AffinityScript cDNA kit (Agilent Technologies, Santa Clara, CA) using oligo(dT) primers according to the manufacturer's protocols. The expression of the OBP and CSP genes in H. elegans was detected by real-time PCR (qRT-PCR) using a Stratagene MX3000 thermocycler (Agilent Technologies, Santa Clara, CA). The cDNA amplification reactions were carried out with $5 \mathrm{ng}$ of total cDNA and a SYBR Fast Universal qPCR kit (Kapa Biosystems, Wilmington, MA). The reaction was performed according to the manufacturer's recommendations. Two technical replicates for each of the three biological replicates were performed, and as a negative control, we used water in the 
196 reaction mixture instead of cDNA. The temperature profile used was $95^{\circ} \mathrm{C}$ for $10 \mathrm{~min}$; followed

197 by 40 cycles of $95^{\circ} \mathrm{C}$ for $15 \mathrm{~s}, 60^{\circ} \mathrm{C}$ for $15 \mathrm{~s}$, and $72{ }^{\circ} \mathrm{C}$ for $20 \mathrm{~s}$. After each amplification step

198 and at the end of the amplification, the fluorescence was measured. For gene expression analysis,

199 specific primers were designed for the OBPs, CSPs (Table 1) and four reference genes (Table 2)

200 from the H. elegans transcriptome. All primers were designed with Beacon Designer v8.13

201 software (Premier Biosoft, Palo Alto, CA, USA). The expression of each gene was normalized to

202 the expression of the EFla gene because a constant expression level was observed for EFla in

203 the different tissues of both sexes, and estimations of relative expression levels were made for

204 each gene using the method of Livak \& Schmittgen (2001). Finally, statistical analysis was

205 performed using analysis of variance (ANOVA) and the multiple range Tukey's test $(\mathrm{p} \leq 0.05)$.

206 All analyses were performed with InfoStat v217.1.2 statistical software (Di Rienzo et al., 2011).

Results

H. elegans reference transcriptome assembly. Using Trinity, filtered reads were assembled into 138,270 contigs ( $N 50$ of 2,189 bp, mean length of $789.3 \mathrm{bp}$ ). Among the assembled contigs, 49,752 (35.98\%) were less than 300 bp in length, 60,126 (43.48\%) contigs were between $300 \mathrm{bp}$ and 1,000 bp in length, and 28,392 (20.53\%) contigs had a size greater than 1,000 bp (Table 3 ).

213 Completeness analysis using BUSCO with the Insecta reference database showed that $97.2 \%$

214 complete conserved genes were found in the assembly and $1.5 \%$ corresponded to fragmented conserved genes, while only $1.3 \%$ of the single-copy ortholog genes were missing (Table 4). An

216 additional BUSCO analysis against Arthropoda and Endopterygota is shown in Table 4, with 217 similar completeness values. 
218 Sequence annotation. The BLASTx alignments revealed that 51,950 contigs $(37.57 \%$ of the

219 total contigs) were annotated with known proteins within the NR database. Most transcript

220 sequences with protein hits matched those of other beetles, such as Oryctes borbonicus and

221 Tribolium castaneum (Fig. 1). Among the BLASTx-aligned contigs, 42,329 (30.61\%) could be

222 annotated based on their sequence homology with GO terms (Fig. 2). Additionally, among the

223 total number of sequences analyzed $(\mathrm{N}=138,270)$ with InterProScan (IPS), 22,889 (16.55\%)

224 sequences were assigned to at least one InterProScan/GO term. The remaining sequences were 225 classified as "IPS match but no GO terms" $(26,881 ; 19.44 \%)$ or as no IPS match $(88,500 ; 64 \%)$

226 (Fig. S1).

227 Chemosensitive protein annotation. The annotation process predicted 23 odorant-binding

228 proteins, one pheromone-binding protein, four chemosensory proteins, 26 gustatory receptors, 70

229 odorant receptors, one odorant receptor coreceptor, three sensory neuron membrane proteins, 15

230 odorant-degrading enzymes, and 22 ionotropic receptors.

231 Quantitative real-time RT-PCR analysis. We investigated the expression patterns of 20

232 putative $H$. elegans OBP and CSP genes by performing a qRT-PCR analysis of the RNA

233 extracted from adult male and female antennae, male and female tibias as well as male and

234 female maxillary palp tissues (Fig. 3). The predicted HelePBP1 in male antennae showed

235 remarkably larger expression than that of the palps and tibias of both sexes but was not

236 distinguishable from that of the female antennae (Fig. 3A). Among the remaining 15 tested

237 OBPs, HeleOBP2, HeleOBP5, HeleOBP8, HeleOBP9 and HeleOBP21 also displayed antenna-

238 specific expression (Fig. 3C, E, G, H, I, P). Indeed, HeleOBP8 and HeleOBP9 expression levels

239 were significantly higher in males than in females (Fig. 3H and I). The HeleOBP5 expression

240 level in the male antennae was similar to that in the female antennae but it was significantly 
241 higher than all other tissues (Fig. 3E). HeleOBP21 expression was similar in the antennae of both

242 sexes, but the expression in female antennae was significantly higher in comparison to all other

243 tissues (Fig. 3P). In addition, HeleOBP4, HeleOBP6, HeleOBP11, HeleOBP13, HeleOBP14,

244 HeleOBP15, HeleOBP17 and HeleOBP18 showed palp-biased expression (Fig. 3D, F, J, K, L,

$245 \mathrm{M}, \mathrm{N}, \mathrm{O})$, with higher expression of HeleOBP14 and HeleOBP15 in males than in females.

246 Interestingly, HeleOBPl showed sex-biased expression, exhibiting higher expression in male

247 palps and antennae (Fig. 3B). Finally, HeleOBP7 showed higher expression in female palps and

248 antennae (Fig. 3G). On the other hand, the expression of CSPs in H. elegans was higher in the

249 palps than in the antennae and tibias (Fig. 3Q-T). Two of the CSPs showed clear sex-biased

250 expression: expression of HeleCSP1 was higher in male palps (Fig. 3Q), while expression levels

251 of HeleCSP3 were significantly higher in female palps than in the other tissues (Fig. 3S). The

252 remaining CSPs HeleCSP2 and HeleCSP4 showed no differences in the expression values for

253 male and female palps (Fig. 3R and T).

254 Phylogenetic analyses of OBPs and CSPs. Clades for different types of OBPs were formed, but

255 no one clade formed exclusively with sequences from a single species (Fig. 4). Among the 18

256 OBP sequences, twelve were classified as Classic, five as Plus-C and one as Dimer. No Minus-C

257 OBPs were identified in the transcriptome. In contrast, the phylogeny for the CSPs (Fig. 5)

258 showed a single clade formed by all four H. elegans CSPs and one sequence from H. oblita.

\section{Discussion}

261 Our study provides the first comprehensive reference transcriptome for Hylamorpha elegans, 262 obtained from antennae, maxillary palps and right hindleg tibia from males and females. The number of unigenes obtained from the current transcriptome were similar to that reported for 
264 Holotrichia parallela and Anomala corpulenta, other agriculturally important scarab beetle pests

265 (Ju et al., 2014; Li et al., 2015; Yi et al., 2018). These numbers also resembled those obtained

266 from the transcriptomes of other beetle species, such as Tomicus yunnanensis (Zhu, Zhao \&

267 Yang, 2012), Ips typographus, Dendroctonus ponderosae (Andersson et al., 2013), Tenebrio

268 molitor (Liu et al., 2015), Brontispa longissima (Bin et al., 2017), Callosobruchus chinensis

269 (Zhang et al., 2017) and Anoplophora chinensis (Sun et al., 2018). Furthermore, BUSCO

270 completeness analysis showed that $98.7 \%$ conserved genes were found in this assembly, while

271 the fraction of missing BUSCOs are quite low (1.3\%). Although a large percentage of genes

272 indeed did not have GO term association assignment (69.39\%), the number of genes with GO

273 term association assignment (30.61\%) is higher compared to percentages of GO annotations

274 reported for antennal transcriptomes in other non-model species $(10.32 \%$ for Phenacoccus

275 solenopsis, 9.77\% for Aenasius bambawalei, (Nie et al., 2018); 13.54\% for Cylas formicarius

276 (Bin et al., 2017). Thus, we conclude that this de novo transcriptome assembly is suitable for

277 both transcript/gene discovery and gene expression analysis (Briones et al., 2018).

From the comparisons of the three tissues studied in both sexes, we identified a higher abundance of all predicted OBP and CSP transcripts in the antennae and/or palps than in the tibia, suggesting that all of these transcripts may take part in chemosensory processes.

Interestingly, the expression levels of several transcripts showed significant differences between males and females. These sex-biased expression patterns suggest that chemosensory genes with higher expression levels in females would play a significant role in host-searching and oviposition behavior (Nie et al., 2018), while chemosensory genes displaying higher expression in males would participate in courtship behavior or female recognition process (Zhou et al., 2014). Besides HelePBP1, HeleOBP8 and HeleOBP9 showed male-biased expression patterns. 
287 (Fig. 3H and I). Similarly, three OBPs in Tenebrio molitor were male antenna-biased, suggesting

288 their role in female recognition (Liu et al., 2015). The ability of OBPs to bind sexual pheromones

289 has been reported in the moth species Amyelois transitella and Spodoptera litura (Liu et al.,

290 2010; Liu, He \& Dong, 2012). These results suggest that further studies of these would reveal

291 their possible role in the sexual behavior of $H$. elegans. Interestingly, among the OBPs,

292 HeleOBP4, $-6,-11,-13,-14,-15,-17$, and -18 were differentially and more expressed in the

293 palps than the other tissues (Fig. 3). While the relative expression of OBPs has been linked to

294 olfaction roles in antennae, in Tenebrio molitor, only eight out of the 19 OBPs were

295 predominantly expressed in the antennae (Liu et al., 2015). Given that insects were collected in

296 the field directly from the foliage of their preferred host (Nothofagus obliqua) where the insects

297 congregate and feed, it is expected that those OBPs could participate in close-range recognition.

298 Another explanation might be the reduced importance of long-range signals in adults' host-

299 seeking process. Both alternatives should be tested in further experiments.

The total number of CSPs found in different insect species varies widely (Pelosi et al., 2014), but the low number of CSPs reported here is in agreement with that reported for the scarab beetle Anomala corpulenta (Li et al., 2015). Further studies, including other non-sensory tissues such as wings and pheromone glands, may increase the quantity of the reported CSPs (Sheng et al., 2017) in addition to studying the expression of CSPs in other developmental stages (Li et al., 2015). However, it is important to note that all four CSPs reported here exhibited palpbiased expression. Moreover, the expression of HeleCSPI was significantly greater in males, suggesting a possible role in sexual recognition. Previous studies of CSPs have shown that they can bind pheromone components in moth species such as Mamestra brassicae (Jacquin-Joly, 2001) and that CSPs are able to bind sex pheromones as well as host-plant volatiles in Sesamia 
310 inferens (Zhang et al., 2014). The expression of HeleCSP3 was significantly greater in female

311 palps (Fig. 3S). Ozaki et al. (2008) assume that female-biased CSP expression is involved in

312 chemoreception and that CSPs transport ligands important to the oviposition behavior of the

313 butterfly Papilio xuthus. Furthermore, CSP-silencing produced significant oviposition reduction

314 in the moth Spodoptera exigua (Gong et al., 2012). Considering these findings, HeleCSP1 and

315 HeleCSP3 could be interesting subjects for further studies to establish their roles in male and

316 female behavior.

317 Phylogenetic analysis for OBPs resulted in clades mostly formed by orthologous

318 sequences grouped by the type of OBP. In H. elegans, most of the OBPs were classified as

319 Classic OBPs, which have been found in all insect species studied (Sánchez-Gracia, Vieira \&

320 Rozas, 2009). Unlike T. castaneum, (Abdel-Latief, 2007) A. corpulenta (Chen et al., 2014) and

321 D. ponderosae (Andersson et al., 2013) H. elegans did not show Minus-C OBPs.

Finally, the five Plus-C sequences were highly divergent (Fig. S2), circumstances that could have arisen by a gene duplication mechanism and rapid evolution, resulting in low sequence identity among the members of this group (Zhou et al., 2004). For CSPs, the

326 the birth-and-death evolutionary model reported for OBP and CSP families (Eirín-López et al., 327 2012), where paralogous genes have higher divergence times than those of orthologous genes 328 (Vieira \& Rozas, 2011). 
331 The current work reports the first transcriptome for a native scarab beetle from Chile and

332 provides information about the annotation of 165 putative proteins related to gustatory and

333 olfactory processes, helping to increase the number of reported sequences for this important

334 insect family. Our initial hypotheses were confirmed: Four out of four studied CSP's transcripts

335 were more abundant in palps, and six out of 16 studied OBP's transcripts were remarkably more

336 abundant in the antennae and eight OBPs were more abundant palps respect to the tissue used as

337 a reference. Additionally, two out of four CSPs and three OBPs showed sex-biased expressions,

338 becoming these transcripts in interesting subjects for further research in Hylamorpha elegans.

\section{References}

341

342

Abdel-Latief M. 2007. A family of chemoreceptors in Tribolium castaneum (Tenebrionidae: Coleoptera). PLoS ONE 2. DOI: 10.1371/journal.pone.0001319.

Aguilera A., Cisternas E., Gerding M., Norambuena H. 1996. Plagas de las praderas. In: Ruiz I. ed. Praderas para Chile. 309-339.

Aguilera A., Guerrero J., Rebolledo R. 2011. Plagas y enfermedades del avellano europeo en La Araucanía. Temuco: Ediciones Universidad de La Frontera.

Altschul SF., Madden TL., Schäffer A., Zhang J., Zhang Z., Miller W., Lipman DJ. 1997. Gapped BLAST and PSI-BLAST: A new generation of protein database search programs. Nucleic Acids Research 25:3389-3402. DOI: 10.1093/nar/25.17.3389.

Andersson MN., Grosse-Wilde E., Keeling CI., Bengtsson JM., Yuen MMS., Li M., Hillbur Y., Bohlmann J., Hansson BS., Schlyter F. 2013. Antennal transcriptome analysis of the 
352

353

354

355

356

357

358

chemosensory gene families in the tree killing bark beetles, Ips typographus and Dendroctonus ponderosae (Coleoptera: Curculionidae: Scolytinae). BMC genomics 14:198. DOI: $10.1186 / 1471-2164-14-198$.

Artigas J. 1994. Entomología económica. Insectos de interés agrícola, forestal, médico y veterinario. Volumen II. Concepción, Chile.

Bauerle P., Rutherford P., Lanfranco D. 1997. Defoliadores de roble (Nothofagus obliqua), raulí (N. alpina), coigüe (N. dombeyi) y lenga (N. pumilio). Bosque 18:97-107.

Bin SY., Qu MQ., Li KM., Peng ZQ., Wu ZZ., Lin JT. 2017. Antennal and abdominal transcriptomes reveal chemosensory gene families in the coconut hispine beetle, Brontispa longissima. Scientific Reports 7:1-13. DOI: 10.1038/s41598-017-03263-1.

Bolger AM., Lohse M., Usadel B. 2014. Trimmomatic: A flexible trimmer for Illumina sequence data. Bioinformatics 30:2114-2120. DOI: 10.1093/bioinformatics/btu170.

Briones C., Nuñez JJ., Pérez M., Espinoza-Rojas D., Molina-Quiroz C., Guiñez R. 2018. De novo male gonad transcriptome draft for the marine mussel Perumytilus purpuratus with a focus on its reproductive-related proteins. Journal of Genomics 6:127-132. DOI: $10.7150 /$ jgen. 27864 .

Brito NF., Moreira MF., Melo ACA. 2016. A look inside odorant-binding proteins in insect chemoreception. Journal of Insect Physiology 95:51-65. DOI: 10.1016/j.jinsphys.2016.09.008.

Chen H., Lin L., Xie M., Zhang G., Su W. 2014. De novo sequencing, assembly and characterization of antennal transcriptome of Anomala corpulenta Motschulsky 
(Coleoptera: Rutelidae). PLoS ONE 9:e114238. DOI: 10.1371/journal.pone.0114238.

374

376

377

378

379

380

381

382

383

384

385

386

387

388

389

390

391

392

393

Cisternas E. 1992. Biología y control de insectos plagas en praderas. In: Seminario Manejo de Praderas Permanentes. Osorno de Chile: INIA-Remehue, 87-117.

Cisternas E. 2002. Seminario Manejo de Praderas Permanentes INIA - Remehue Osorno. Seminario Manejo de Praderas Permanentes INIA - Remehue Osorno :87-117.

Cisternas E. 2013. Insectos plaga de importancia económica asociados al arándano. In: Undurraga P, Vargas S. eds. Manual de arándano. Boletín INIA N²63. Instituto de Investigaciones Agropecuarias, 91-106.

Conesa A., Götz S. 2008. Blast2GO: A comprehensive suite for functional analysis in plant genomics. International Journal of Plant Genomics 2008. DOI: 10.1155/2008/619832.

Dahanukar A., Hallem E a., Carlson JR. 2005. Insect chemoreception. Current Opinion in Neurobiology 15:423-430. DOI: 10.1016/j.conb.2005.06.001.

Durán L. 1951. Aspectos ecológicos de la biología del sanjuan verde, Hylamorpha elegans (Burm.) y mención de las demás especies de escarabeidos perjudiciales en Cautín. Agricultura Técnica 12:24-36.

Eirín-López J., Rebordino L., Rooner A.P., Rozas J. 2012. The birth- and- death evolution of multigene. Genome Dynamics Basel 7:170-196. DOI: 10.1159/000337119

Gadenne C., Barrozo RB., Anton S. 2016. Plasticity in insect olfaction: to smell or not to smell? Annual Review of Entomology 61:317-333. DOI: 10.1146/annurev-ento-010715-023523.

Glaser N., Gallot A., Legeai F., Montagné N., Poivet E., Harry M., Calatayud PA., Jacquin-Joly E. 2013. Candidate chemosensory genes in the stemborer Sesamia nonagrioides. 
395 Gong L., Luo Q., Rizwan-Ul-Haq M., Hu MY. 2012. Cloning and characterization of three 396 chemosensory proteins from Spodoptera exigua and effects of gene silencing on female 397 survival and reproduction. Bulletin of Entomological Research 102:600-609. DOI: $10.1017 / \mathrm{S} 0007485312000168$.

399

400

401

402

403

404 405

406

407

408

409

410

411

412

413

414

415

González-González, A., Palma-Millanao, R., Yáñez, O., Rojas, M., Mutis, A., Venthur, H.,Quiroz, A, Ramírez, C. C. 2016. Virtual screening of plant volatile compounds reveals a high affinity of Hylamorpha elegans (Coleoptera: Scarabaeidae) odorant-binding proteins for sesquiterpenes from its native host. Journal of Insect Science, 16: 30. DOI: 10.1093/jisesa/iew008.

Gurevich A., Saveliev V., Vyahhi N., Tesler G. 2013. QUAST: Quality assessment tool for genome assemblies. Bioinformatics 29:1072-1075. DOI: 10.1093/bioinformatics/btt086.

Haas BJ., Papanicolaou A., Yassour M., Grabherr M., Blood PD., Bowden J., Couger MB., Eccles D., Li B., Lieber M., Macmanes MD., Ott M., Orvis J., Pochet N., Strozzi F., Weeks N., Westerman R., William T., Dewey CN., Henschel R., Leduc RD., Friedman N., Regev A. 2013. De novo transcript sequence reconstruction from RNA-seq using the Trinity platform for reference generation and analysis. Nature protocols 8:1494-512. DOI: $10.1038 /$ nprot.2013.084.

Iatrou K., Biessmann H. 2008. Sex-biased expression of odorant receptors in antennae and palps of the African malaria vector Anopheles gambiae. Insect Biochemistry and Molecular Biology 38:268-274. DOI: 10.1016/j.ibmb.2007.11.008.

Jackson T a., Klein MG. 2006. Scarabs as pests: a continuing problem. The Coleopterists 
417 Jacquin-Joly E. 2001. Functional and expression pattern analysis of chemosensory proteins 418 expressed in antennae and pheromonal gland of Mamestra brassicae. Chemical Senses 26:833-844. DOI: 10.1093/chemse/26.7.833.

420

421

422

423

424

425

426

427

428

429

430

431

432

433

434

435

436

Ju Q., Li X., Jiang X-J., Qu M-J., Guo X-Q., Han Z-J., Li F. 2014. Trancriptome and tissuespecific expression analysis of OBP and CSP genes in the dark black chafer. Archives of Insect Biochemistry and Physiology 87:177-200. DOI: 10.1002/arch.21188.

Lanfranco D., Rojas E., Rios R., Ruiz C. 2001. Insect defoliators of Nothofagus obliqua (Roble) in South Chile: Two years monitoring species and their damage. Integrated Management and Dynamics of Forest Defoliating Insects, Proceedings 277:91-103.

Langmead B., Trapnell C., Pop M., Salzberg SL. 2009. Ultrafast and memory-efficient alignment of short DNA sequences to the human genome. Genome biology 10:R25. DOI: 10.1186/gb2009-10-3-r25.

Leal WS. 2013. Odorant reception in insects : roles of receptors, binding proteins, and degrading enzymes. Annual Review of Entomology 58:373-391. DOI: 10.1146/annurev-ento-120811153635.

Leal WS. 2014. Deciphering the rosetta stone of insect chemical communication. American Entomologist 60:223-230. DOI: 10.1093/ae/60.4.223

Lefort M-C., Boyer S., De Romans S., Glare T., Armstrong K., Worner S. 2014. Invasion success of a scarab beetle within its native range: host range expansion versus host-shift. PeerJ 2:e262. DOI: 10.7717/peerj.262. 
437 Lefort MC., Worner SP., Rostas M., Vereijssen J., Boyer S. 2015. Responding positively to plant

438 defences, a candidate key trait for invasion success in the New Zealand grass grub

439 Costelytra zealandica. New Zealand Journal of Ecology 39:128-132.

440

Li X., Ju Q., Jie W., Li F., Jiang X., Hu J., Qu M. 2015. Chemosensory gene families in adult antennae of Anomala corpulenta Motschulsky (Coleoptera: Scarabaeidae: Rutelinae). PLoS ONE 10:e0121504. DOI: 10.1371/journal.pone.0121504.

Li H., Zhang L., Ni C., Shang H., Zhuang S., Li J. 2013. Molecular recognition of floral volatile with two olfactory related proteins in the Eastern honeybee (Apis cerana). International Journal of Biological Macromolecules 56:114-121. DOI: 10.1016/j.ijbiomac.2013.01.032.

Liu NY., He P., Dong SL. 2012. Binding properties of pheromone-binding protein 1 from the common cutworm Spodoptera litura. Comparative Biochemistry and Physiology - B Biochemistry and Molecular Biology 161:295-302. DOI: 10.1016/j.cbpb.2011.11.007.

Liu S., Rao XJ., Li MY., Feng MF., He MZ., Li SG. 2015. Identification of candidate chemosensory genes in the antennal transcriptome of Tenebrio molitor (Coleoptera: Tenebrionidae). Comparative Biochemistry and Physiology - Part D: Genomics and Proteomics 13:44-51. DOI: 10.1016/j.cbd.2015.01.004.

Liu Z., Vidal DM., Syed Z., Ishida Y., Leal WS. 2010. Pheromone binding to general odorantbinding proteins from the navel orangeworm. Journal of Chemical Ecology 36:787-794. DOI: $10.1007 / \mathrm{s} 10886-010-9811-5$.

Livak KJ., Schmittgen TD. 2001. Analysis of relative gene expression data using real-time quantitative PCR and the $2-\Delta \Delta \mathrm{CT}$ method. Methods 408:402-408. DOI: 
459 Mao A., Zhou J., Bin Mao., Zheng Y., Wang Y., Li D., Wang P., Liu K., Wang X., Ai H. 2016.

460 Sex pheromone recognition and characterization of three pheromone-binding proteins in the

461 legume pod borer, Maruca vitrata Fabricius (Lepidoptera: Crambidae). Scientific Reports

$462 \quad 6: 34484$. DOI: $10.1038 / \operatorname{srep} 34484$.

463 Missbach C., Dweck HKM., Vogel H., Vilcinskas A., Stensmyr MC., Hansson BS., Grosse-

464 wilde E. 2014. Evolution of insect olfactory receptors. eLife 3:e02115. DOI:

465 10.7554/eLife.02115.

466

467

468

469

470

471

472

473

474

475

476

477

478

479

480

Moreton J., Izquierdo A., Emes RD. 2016. Assembly, assessment, and availability of De novo generated eukaryotic transcriptomes. Frontiers in Genetics 6:1-9. DOI: 10.3389/fgene.2015.00361.

Mutis A., Palma R., Parra L., Alvear M., Isaacs R., Morón M., Quiroz A. 2014. Morphology and Distribution of Sensilla on the Antennae of Hylamorpha elegans Burmeister (Coleoptera: Scarabaeidae). Neotropical Entomology 43:260-265. DOI: 10.1007/s13744-014-0208-y.

Nie XP., Li QL., Xu C., Li DZ., Zhang Z., Wang M-Q., Zhou AM., Li SQ. 2018. Antennal transcriptome and odorant binding protein expression profiles of an invasive mealybug and its parasitoid. Journal of Applied Entomology 142:149-161. DOI: 10.1111/jen.12417.

Ozaki K., Utoguchi A., Yamada A., Yoshikawa H. 2008. Identification and genomic structure of chemosensory proteins (CSP) and odorant binding proteins (OBP) genes expressed in foreleg tarsi of the swallowtail butterfly Papilio xuthus. Insect Biochemistry and Molecular Biology 38:969-976. DOI: 10.1016/j.ibmb.2008.07.010.

Pelosi P., Iovinella I., Felicioli A., Dani FR., Kadarkarai ME. 2014. Soluble proteins of chemical communication : an overview across arthropods. Frontiers in Physiology 5:1-14. DOI: 
482

483

484

485

486

487

488

489

490

491

492

493

494

495

496

497

498

499

500

501

Pelosi P., Iovinella I., Zhu J., Wang G., Dani FR. 2018. Beyond chemoreception: diverse tasks of soluble olfactory proteins in insects. Biological Reviews 93:184-200. DOI: 10.1111/brv.12339.

Quiroz A., Palma R., Etcheverría P., Navarro V., Rebolledo R. 2007. Males of Hylamorpha elegans Burmeister (Coleoptera: Scarabaeidae) are attracted to odors released from conspecific females. Environmental Entomology 36:272-280. DOI: 10.1603/0046225X(2007)36[272:MOHEBC]2.0.CO;2.

Ratcliffe BC., Ocampo FC. 2002. A review of the genus Hylamorpha Arrow (Coleoptera: Scarabaeidae: Rutelinae: Anoplognathini: Brachysternina). The Coleopterists Bulletin 56:367-378. DOI: 10.1649/0010-065X(2002)056[0367:AROTGH]2.0.CO;2.

Di Rienzo JA., Casanoves F., Balzarini MG., González L., Tablada M., Robledo y CW. 2011. InfoStat versión 2011. Grupo InfoStat, FCA, Universidad Nacional de Córdoba, Argentina. 8:195-199.

Sánchez-Gracia A., Vieira FG., Rozas J. 2009. Molecular evolution of the major chemosensory gene families in insects. Heredity 103:208-216. DOI: 10.1038/hdy.2009.55.

Sheng S., Liao CW., Zheng Y., Zhou Y., Xu Y., Song WM., He P., Zhang J., Wu FA. 2017. Candidate chemosensory genes identified in the endoparasitoid Meteorus pulchricornis (Hymenoptera: Braconidae) by antennal transcriptome analysis. Comparative Biochemistry and Physiology - Part D: Genomics and Proteomics 22:20-31. DOI: 10.1016/j.cbd.2017.01.002. 
502 Simão FA., Waterhouse RM., Ioannidis P., Kriventseva E V., Zdobnov EM. 2015. BUSCO:

503 Assessing genome assembly and annotation completeness with single-copy orthologs.

504 Bioinformatics 31:3210-3212. DOI: 10.1093/bioinformatics/btv351.

505 Spinelli S., Lagarde A., Iovinella I., Legrand P., Tegoni M., Pelosi P., Cambillau C. 2012.

506 Crystal structure of Apis mellifera OBP14, a C-minus odorant-binding protein , and its 507 complexes with odorant molecules. Insect Biochemistry and Molecular Biology 42:41-50.

508 DOI: $10.1016 /$ j.ibmb.2011.10.005.

509 Stengl M., Funk NW. 2013. The role of the coreceptor Orco in insect olfactory transduction.

510 Journal of Comparative Physiology A: Neuroethology, Sensory, Neural, and Behavioral

511 Physiology 199:897-909. DOI: 10.1007/s00359-013-0837-3.

512 Suh E., Bohbot JD., Zwiebel LJ. 2014. Peripheral olfactory signaling in insects. Current Opinion 513 in Insect Science 6:86-92. DOI: 10.1016/j.cois.2014.10.006.

514 Sun L., Zhang YN., Qian JL., Kang K., Zhang XQ., Deng JD., Tang YP., Chen C., Hansen L., 515 Xu T., Zhang QH., Zhang LW. 2018. Identification and expression patterns of Anoplophora 516 chinensis (Forster) Chemosensory receptor genes from the antennal transcriptome. 517 Frontiers in Physiology 9:1-13. DOI: 10.3389/fphys.2018.00090.

518 Thompson JD., Higgins DG., Gibson TJ. 1994. CLUSTAL W : improving the sensitivity of 519 progressive multiple sequence alignment through sequence weighting, position-specific gap 520 penalties and weight matrix choice. $22: 4673-4680$.

521 Venthur H., Mutis A., Zhou J-J., Quiroz A. 2014. Ligand binding and homology modelling of 522 insect odorant-binding proteins. Physiological Entomology 39:183-198. DOI: 10.1111/phen.12066. 
524 Venthur H., Zhou J-J., Mutis A., Ceballos R., Mella-Herrera R., Larama G., Avila A., Iturriaga-

525 Vásquez P., Faundez-Parraguez M., Alvear M., Quiroz A. 2016. $\beta$-Ionone as putative

526 semiochemical suggested by ligand binding on an odorant-binding protein of Hylamorpha

527 elegans and electroantennographic recordings. Entomological Science. DOI:

$528 \quad 10.1111 /$ ens. 12180.

529 Vieira FG., Rozas J. 2011. Comparative genomics of the odorant-binding and chemosensory

530 protein gene families across the arthropoda: Origin and evolutionary history of the

531 chemosensory system. Genome Biology and Evolution 3:476-490. DOI:

$532 \quad 10.1093 /$ gbe/evr033.

533 Yi JK., Yang S., Wang S., Wang J., Zhang XX., Liu Y., Xi JH. 2018. Identification of candidate

534 chemosensory receptors in the antennal transcriptome of the large black chafer Holotrichia

535 parallela Motschulsky (Coleoptera: Scarabaeidae). Comparative Biochemistry and

536 Physiology - Part D: Genomics and Proteomics 28:63-71. DOI: 10.1016/j.cbd.2018.06.005.

537 Zhang YN., Kang K., Xu L., Zhu XY., Qian JL., Zhang ZJ., He P., Li XM. 2017. Deep

538 sequencing of antennal transcriptome from Callosobruchus chinensis to characterize

539 odorant binding protein and chemosensory protein genes. Journal of Stored Products

$540 \quad$ Research 74:13-21. DOI: 10.1016/j.jspr.2017.08.006.

541 Zhang J., Walker WB., Wang G. 2015. Pheromone reception in moths: From molecules to

542 behaviors. Progress in Molecular Biology and Translational Science. 130: 109-128. DOI:

$543 \quad 10.1016 /$ bs.pmbts.2014.11.005.

544 Zhang YN., Ye ZF., Yang K., Dong SL. 2014. Antenna-predominant and male-biased CSP19 of

545 Sesamia inferens is able to bind the female sex pheromones and host plant volatiles. Gene 
547 Zhou JJ., Huang W., Zhang GA., Pickett JA., Field LM. 2004. "Plus-C” odorant-binding protein 548 genes in two Drosophila species and the malaria mosquito Anopheles gambiae. Gene 327:117-129. DOI: 10.1016/j.gene.2003.11.007.

550

551

552

553

554

555

556

557

558

559

560

561
Zhou SS., Sun Z., Ma W., Chen W., Wang MQ. 2014. De novo analysis of the Nilaparvata lugens (Stål) antenna transcriptome and expression patterns of olfactory genes. Comparative Biochemistry and Physiology - Part D: Genomics and Proteomics 9:31-39. DOI: 10.1016/j.cbd.2013.12.002.

Zhou Y Le., Zhu XQ., Gu SH., Cui H huan., Guo YY., Zhou JJ., Zhang YJ. 2014. Silencing in Apolygus lucorum of the olfactory coreceptor Orco gene by RNA interference induces EAG response declining to two putative semiochemicals. Journal of Insect Physiology 60:31-39. DOI: $10.1016 /$ j.jinsphys.2013.10.006.

Zhu JY., Zhao N., Yang B. 2012. Global transcriptional analysis of olfactory genes in the head of pine shoot beetle, Tomicus yunnanensis. Comparative and Functional Genomics 2012. DOI: $10.1155 / 2012 / 491748$. 


\section{Table 1 (on next page)}

Identification, sequence length, primers sequences, and amplicon size of OBPs, CSPs, and PBP genes to Hylamorpha elegans. 
Identification, sequence length, primers sequences, and amplicon size of OBPs, CSPs, and PBP genes to Hylamorpha elegans.

\begin{tabular}{lcccc}
\hline Sequence ID & $\begin{array}{c}\text { Sequence } \\
\text { Length }(\mathrm{pb})\end{array}$ & Forware primer sequences $\left(5^{\prime}-3\right)$ & Reverse primer sequences $\left(5^{\prime}-3\right)^{\prime}$ & $\begin{array}{c}\text { Product } \\
\text { Length }(\mathrm{pb})\end{array}$ \\
\hline \hline
\end{tabular}

\section{a) $O B P s$}

$\begin{array}{lll}\text { HePBP1 } & 426 & \text { TGATGATGGTATTATAGATG } \\ \text { HeleOBP1 } & 432 & \text { AGACAGACAGGAACGGATA } \\ \text { HeleOBP2 } & 477 & \text { AAGTGCTGGTGCTACTAA } \\ \text { HeleOBP3 } & 552 & \text { GCTGGATGTGTTTAAGTTA } \\ \text { HeleOBP4 } & 450 & \text { GTGGCATACTGAATAGCA } \\ \text { HeleOBP5 } & 501 & \text { GCAAGACAATAACGGTAA } \\ \text { HeleOBP6 } & 449 & \text { TACTTGCTTCACTTCTCC } \\ \text { HeleOBP7 } & 507 & \text { GCTTAGAATCATCCACAA } \\ \text { HeleOBP8 } & 453 & \text { GTTACCAGGATACAAGAAG } \\ \text { HeleOBP9 } & 453 & \text { TGGAAGCAGATAGCGATTA } \\ \text { HeleOBP11 } & 471 & \text { CCTTGCCTTGTGCTCATA } \\ \text { HeleOBP13 } & 414 & \text { AATCGCTACCAGGAACAG } \\ \text { HeleOBP14 } & 693 & \text { AAGAAGGAGATGAGATGTG } \\ \text { HeleOBP15 } & 633 & \text { GCTCAATCGTTAGAATGT } \\ \text { HeleOBP17 } & 447 & \text { ATCAAATAACGAACCCTCTC } \\ \text { HeleOBP18 } & 468 & \text { ATAGTGGACGCCGTTATG } \\ \text { HeleOBP19 } & 384 & \text { TCTCATAAGTGTGCGAAT } \\ \text { HeleOBP21 } & 927 & \text { TACGAGATGAAGGCGAAT }\end{array}$

GGTAGACATTATCACAAG 128 TATCTATAAGGGTCGGGTCAA 77 GGTCCTTCATCGCTTCTA 167 CGAATCTGCGTTGTAATG 92

TAGGTCTGTTGACAAGGA 124 CACGGTATCTAAGCAGTA 121 GGTATTCATCGCTTGTTG 80 ATGCCTATACTTCGTAGA 157 CGACTAGATTCCGTAGAT 103 GTCATCATCATCAGGAACAG 80 CCTGTGTCCATAATCTTCTCTAA 84 GTGACGCATACCGACATT 100 AGCCTAAATCAGCAGTAAT 124 TGTGCTTCATCTTCATAAG 161 CCGCTGCTATTCAGTATG 81 CTGCCTGTTAGACCTTGAC $\quad 77$ CGTAACTCCTTCCGTATA 83 AATTGGCTGTAGGTGTAAG 88

b) $\mathrm{CSPS}$

HeleCSP 1 HeleCSP2 HeleCSP3 405 390 390 HeleCSP4
TTGTTGTGTTAAGTGTTGT GGTGTTAGTTGTGTTAAGTGTT GTGTTAGTTGTGTTGAGT AGTATTGTCGTGGTAGTG
CCAGAAGACGATCATTATTC CCTTCCAGAAGACGATCATTA CTTATTGTATCCTTCCAGAA GCATGGTCCTTCATCTAA

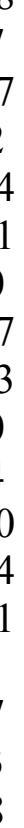

114

123

132

141 


\section{Table 2 (on next page)}

Primer sequences and amplicon sizes for the normalizer genes tested in Hylamorpha elegans. 
1 Table 2:

2 Primers sequences, and amplicon size for normalizer genes tested in Hylamorpha elegans.

\begin{tabular}{|c|c|c|c|}
\hline Normalized Gene ID & Forward primer sequences $\left(5^{\prime}-3\right)$ & Reverse primer sequences $\left(5^{\prime}-3\right)$ & Product Length (pb) \\
\hline $\mathrm{Hel}$ & CGA & GC & 75 \\
\hline HeEFla & TCAAGCAACTTATTGTAGGT & TTAATGTACGACGAGACTTC & 103 \\
\hline HeB-Actin & СТСТТССААССТТСАТТСТT & GTCAACATCGCACTTCAT & 84 \\
\hline$H e G A D P H$ & AATATCTACCAGGACATAA & ATGAGTATATCTGCTTCT & 85 \\
\hline
\end{tabular}


Table 3 (on next page)

H. elegans transcriptome assembly statistics. 


\section{Table 3:}

2

3 Transcriptome assembly statistics

4

\begin{tabular}{ll}
\hline H. elegans transcriptome assembly statistics \\
\hline \# contigs $(>=0 \mathrm{bp})$ & 138,270 \\
\# contigs $(>=1000 \mathrm{bp})$ & 28,392 \\
Total length $(>=0 \mathrm{bp})$ & $109,137,411$ \\
Total length $(>=1000 \mathrm{bp})$ & $66,930,846$ \\
\# contigs & 51,549 \\
Largest contig & 27,382 \\
Total length & $82,968,309$ \\
GC $(\%)$ & 36.85 \\
N50 & 2,189 \\
N75 & 1,196 \\
\# N's per $100 \mathrm{kbp}$ & 0 \\
H. elegans transcriptome assembly statistics \\
\# contigs ( $>=0$ bp) & 138,270 \\
\# contigs ( $>=1000 \mathrm{bp})$ & 28,392 \\
Total length $(>=0$ bp) & $109,137,411$ \\
Total length $(>=1000$ bp) & $66,930,846$ \\
\# contigs & 51,549 \\
Largest contig & 27,382 \\
Total length & $82,968,309$ \\
GC (\%) & 36.85 \\
N50 & 2,189 \\
N75 & 1,196 \\
\# N's per $100 \mathrm{kbp}$ & 0 \\
\hline
\end{tabular}


Table 4 (on next page)

BUSCO analysis of the $H$. elegans assembly. 
1 Table 4:

2

3 BUSCO analysis on $\boldsymbol{H}$. elegans assembly.

4

\begin{tabular}{lccc}
\hline \multicolumn{1}{c}{ BUSCO category } & Insecta & Endopterygota & Arthropoda \\
\hline Complete BUSCOs (C) & $1611(97.2 \%)$ & $2320(95 \%)$ & $1044(98 \%)$ \\
Complete and single-copy BUSCOs (S) & $1129(68.1 \%)$ & $1573(64.4 \%)$ & $746(70 \%)$ \\
Complete and duplicated BUSCOs (D) & $482(29.1 \%)$ & $747(30.6 \%)$ & $298(28 \%)$ \\
Fragmented BUSCOs (F) & $25(1.5 \%)$ & $67(2.7 \%)$ & $13(1.2 \%)$ \\
Missing BUSCOs (M) & $22(1.3 \%)$ & $55(2.3 \%)$ & $9(0.8 \%)$ \\
Total BUSCO groups searched & $1658(100 \%)$ & $2442(100 \%)$ & $1066(100 \%)$
\end{tabular}

5

6 


\section{Figure 1}

Species distribution of unigene sequences of $H$. elegans transcripts relative to other species using homologous BLASTx hits and the NR-NCBI database

\section{Species Distribution}

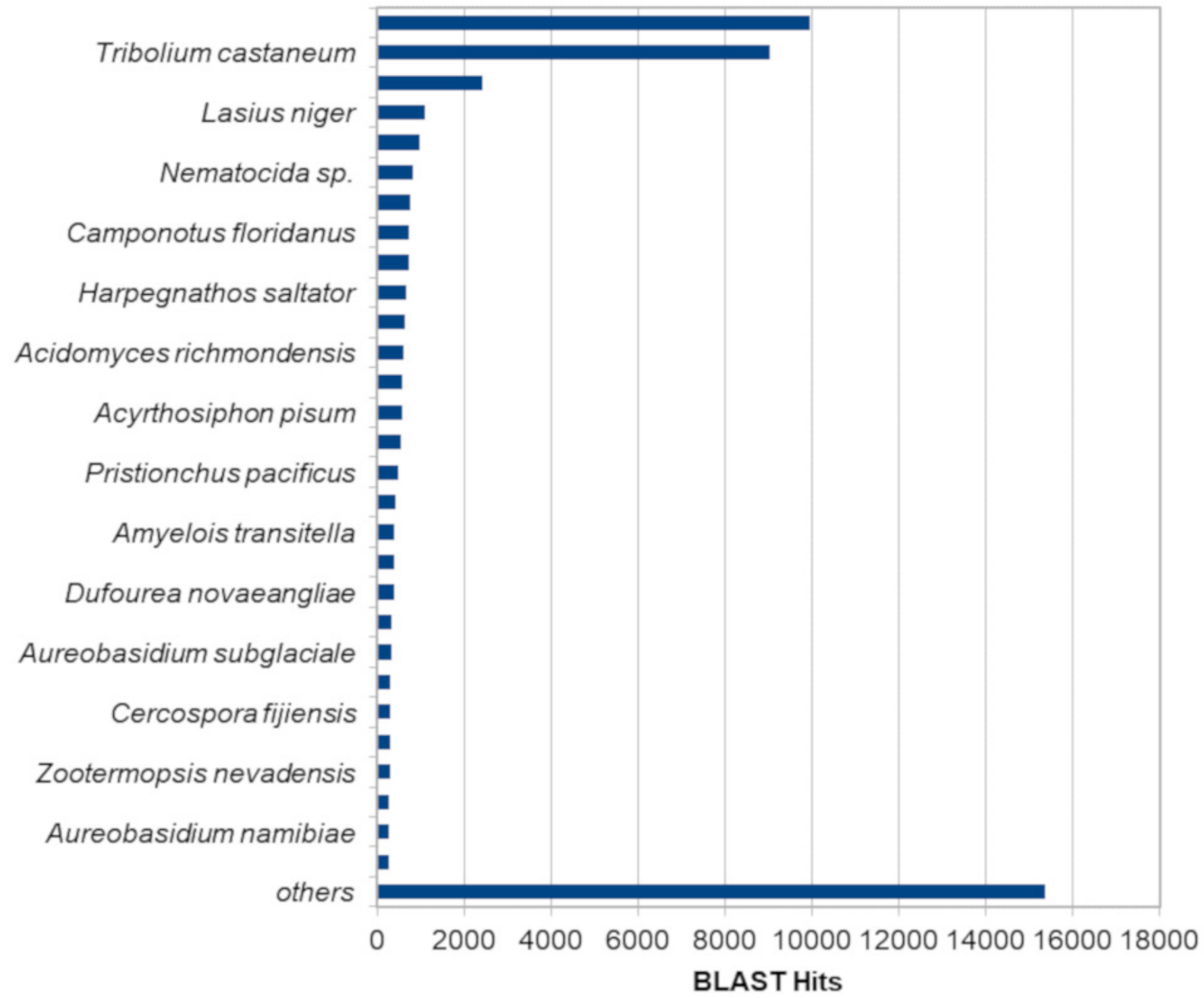




\section{Figure 2}

Pie chart for the distribution of the Blast2GO annotations.

\section{Annotation Distribution}

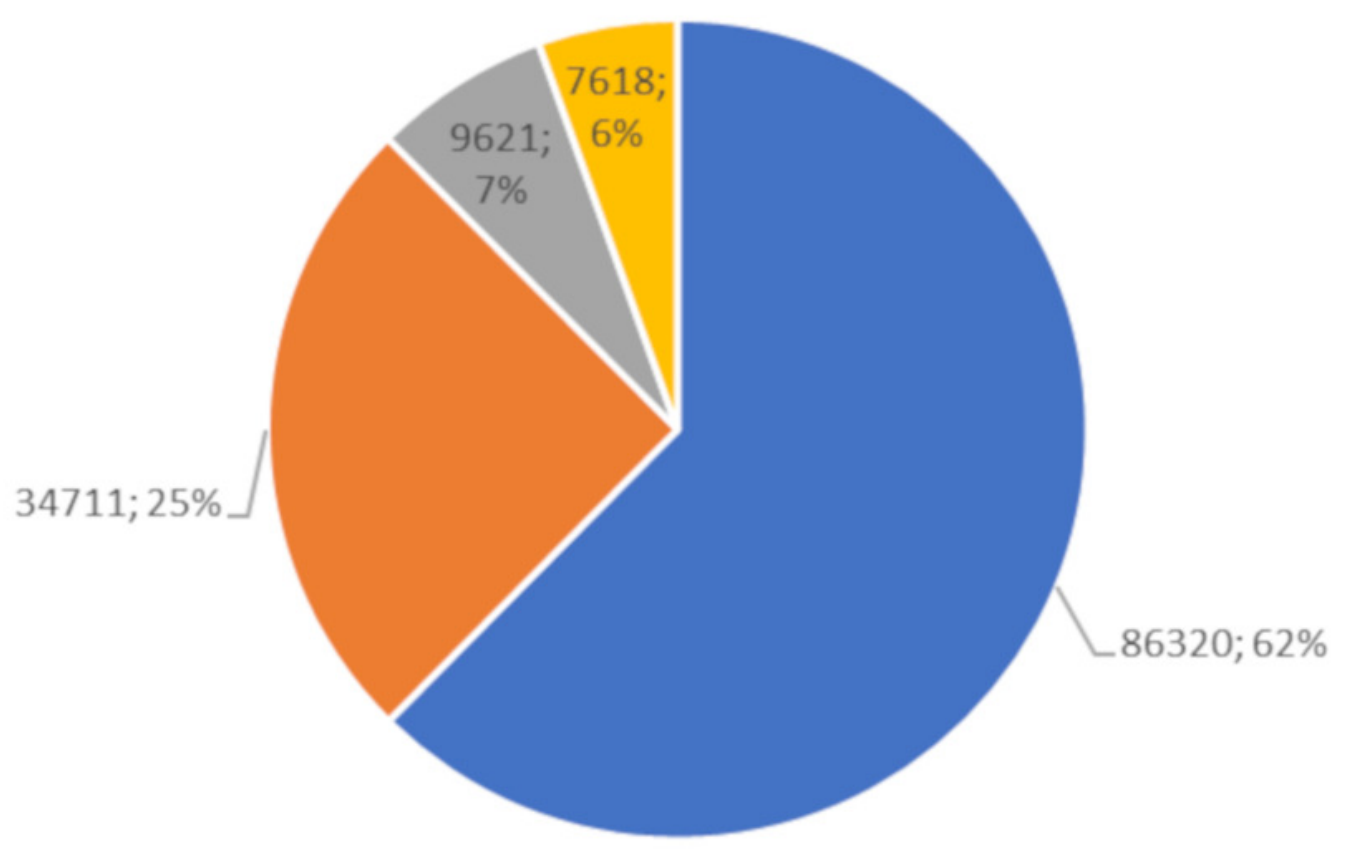

- Blasted without Hits = GOSlim = With Blast Hits = With GO Mapping 


\section{Figure 3}

Relative expression levels of the putative chemosensory transcripts of $H$. elegans using qRT-PCR.

(A) H. elegans PBP1; (B-P) H. elegans OBPs; (Q-T) H. elegans CSPs. The x-axis shows the different tissues. The $y$-axis shows the relative expression level. TM, male tibias; TF, female tibias; AM, male antennae; AF, female antennae; TM, male palps; PF, female palps. Different letters indicate differences according to Tukey-HSD test $(p \leq 0.05)$. 

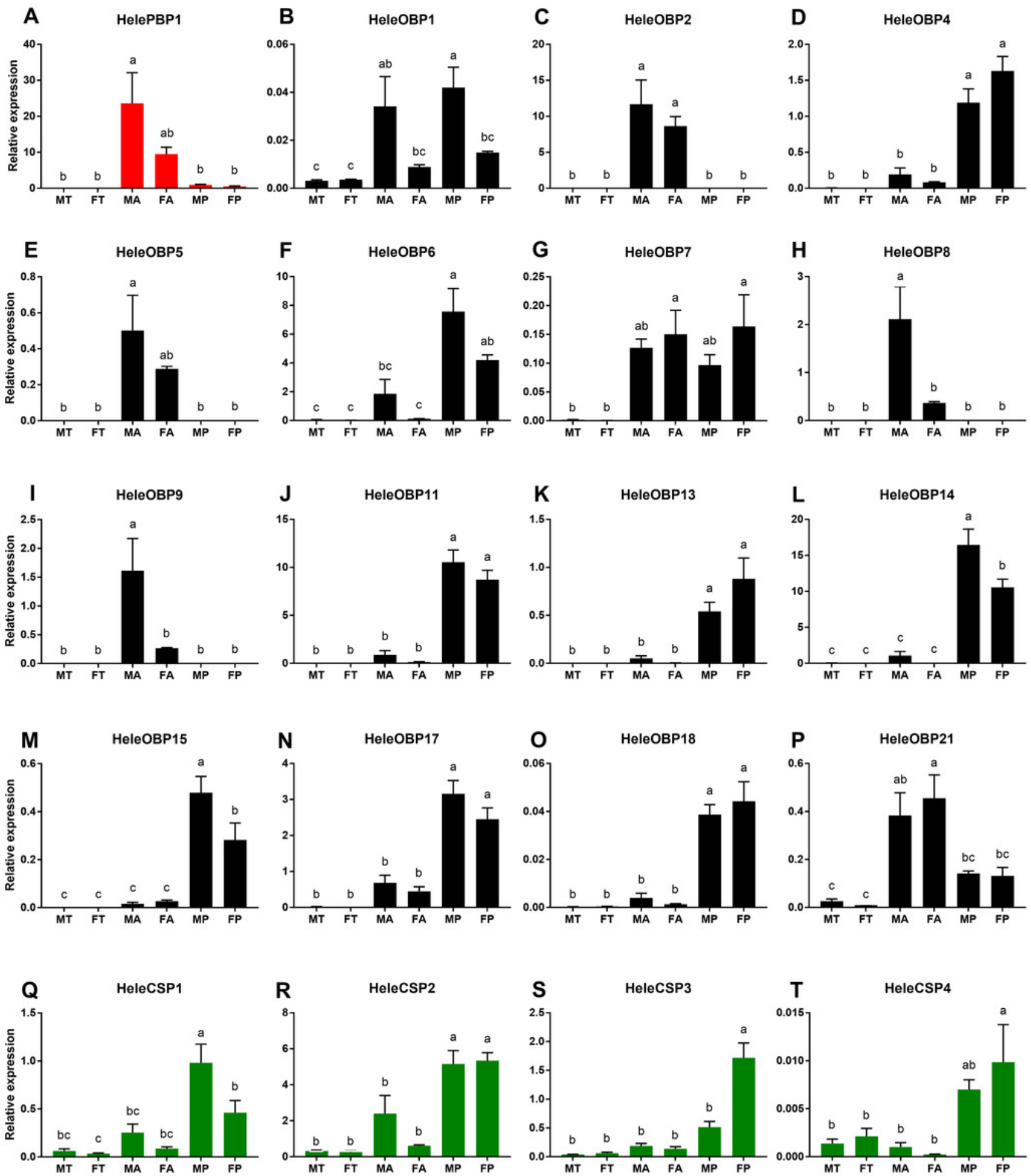
Figure 4

Phylogenetic relationship of candidate OBPs from $\mathrm{H}$. elegans and other coleopteran species.

The tree was built using the maximum-likelihood method with CLC Workbench v7.8.1 with 1,000 replications. Different color arches indicate different types of OBP classification. Different letter colors indicate the species. OBP names for species other than $\mathrm{H}$. elegans are assigned by NCBI ID numbers. Reference line indicates tree scale. 


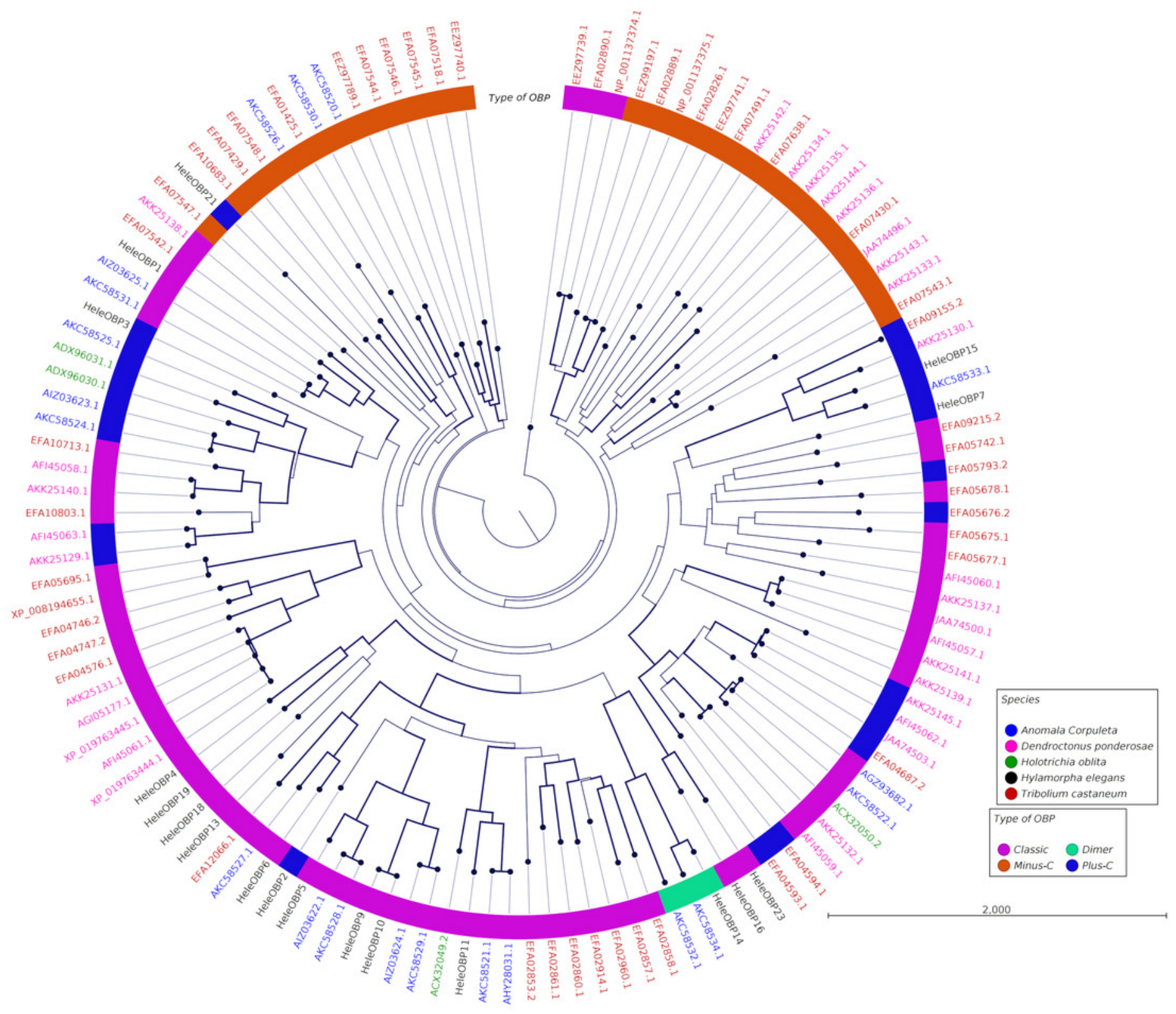




\section{Figure 5}

Phylogenetic relationship of candidate CSPs from $\mathrm{H}$. elegans and other coleopteran species.

The tree was built using the maximum-likelihood method with CLC Workbench v7.8.1 with 1,000 replications. Different color arches indicate different types of OBP classification. Different letter colors indicate the species. OBP names for other species than $H$. elegans are assigned by NCBI ID numbers. Reference line indicates tree scale.

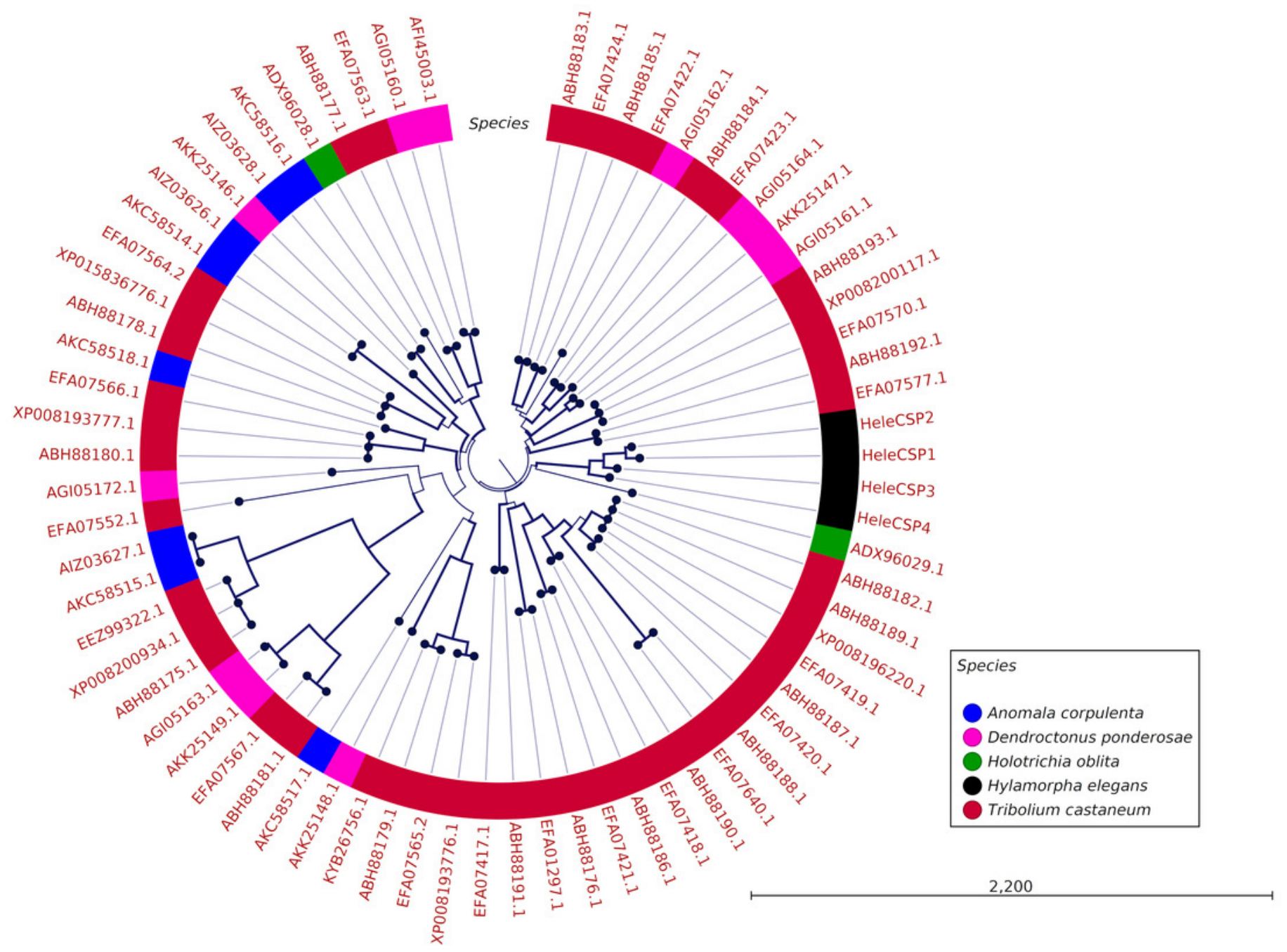

